\title{
Avaliação da autonomia funcional em idosos comunitários
}

\author{
Assessment of functional autonomy in the elderly community
}

Autonomia funcional em idosos

\begin{abstract}
Bruna Raphaela Santos Abreu1, Adriana Sousa Regoํㄹ Aldiléa Mota de Azevedo Vieira², José Newton Lacet Vieira ${ }^{3}$
\end{abstract}

Resumo: Objetivo: Avaliar a autonomia funcional em idosos comunitários. Materiais e métodos: Caracterizouse como uma pesquisa de campo do tipo descritiva, observacional, e transversal, realizada com 34 idosos comunitários com idade igual ou superior a 60 anos, de ambos os sexos, na União de Moradores do bairro Sol e Mar, do município de São Luís. Foi utilizado o Questionário validado GDLAM. O presente estudo foi aprovado pelo Comitê de Ética em Pesquisa em Humanos (CEP), da Universidade CEUMA, sob parecer no 289.169/2013. Os dados foram digitados e analisados no SPSS 18.0. Na estatística descritiva, as variáveis qualitativas são apresentadas através de frequências absolutas e relativas. Para comparar a autonomia funcional por sexo e faixa etária foi utilizado o Qui-quadrado sendo considerado significativo $p \leq 0,05$. Resultados: Foi verificado que $85,3 \%$ (29) dos idosos apresentam autonomia fraca. Na comparação da autonomia por sexo verificou-se que entre as idosas $83,9 \%$ apresentam autonomia fraca e entre os homens $100 \%$. Com relação à faixa etária, embora tenha sido observado que $89,5 \%$ dos idosos com idade entre 60 e 70 anos tenham autonomia fraca e aqueles com idade entre 71 e 81 anos apenas $80,0 \%$ tenham autonomia fraca. Conclusão: Concluiu-se que a grande maioria da amostra estudada apresentou níveis baixos de autonomia funcional, em especial nos idosos com menor faixa etária.

Palavras-chave: Autonomia; Participação da comunidade; Idoso.

\begin{abstract}
Objective: To evaluate the functional autonomy in the elderly community. Materials and methods: It was characterized as a descriptive, observational, and cross-sectional field survey conducted with 34 community-dwelling elderly individuals of 60 years and older, of both genders, in the Union of Residents of the Sol e Mar neighborhood of the municipality of São Luís. The validated GDLAM Questionnaire was used. The present study was approved by the Committee on Ethics in Human Research (CEP), CEUMA University, under opinion $n^{\circ}$ 289.169/2013. Data were entered and analyzed in SPSS 18.0. In descriptive statistics, the qualitative variables are presented through absolute and relative frequencies. In order to compare the functional autonomy by sex and age group, chi-square was used, being considered significant $p \leq 0.05$. Results: It was verified that $85.3 \%$ (29) of the elderly had weak autonomy. In the comparison of the autonomy by sex, it was verified that among the elderly women, $83.9 \%$ had weak autonomy and $100 \%$ among the men. Regarding the age group, although it was observed that $89.5 \%$ of the elderly with age between 60 and 70 years had weak autonomy and those with age between 71 and 81 years old only $80.0 \%$ had weak autonomy. Conclusion: It was concluded that the great majority of the studied sample presented low levels of functional autonomy, especially in the elderly with lower age group.
\end{abstract}

Keywords: Autonomy; Community participation; Old man

\footnotetext{
1 Departamento de Fisioterapia. Universidade Ceuma

2 Fisioterapeuta. Espaço Fisiofuncional

${ }^{3}$ Departamento de Fisioterapia. Universidade Ceuma. Endereço para correspondência: Avenida Nova lorque, Q-5 E, C-03, Central Parque, Araçagy, São José de Ribamar-MA. CEP: 65.110-000. Fone: (98) 981109633.
} 


\section{Introdução}

Segundo Barbosa et al ${ }^{1}$ devido ao avanço da expectativa de vida, e com o crescente aumento de estudos que buscam promover a melhoria na qualidade de vida dos idosos pode-se inferir que a prevalência de idosos com autonomia funcional cresce e cada vez mais os idosos buscam autonomia para realizarem suas atividades do cotidiano. Para que se realize uma tarefa vários sistemas são envolvidos como 0 nervoso e 0 musculoesquelético, geralmente idosos com mais ou menos 80 anos, encontramse com esses sistemas fragilizados, evitando ou limitando a realização de suas atividades, sendo assim, aumentando a incapacidade funcional gradualmente ${ }^{2}$.

Segundo Gonçalves et al ${ }^{3}$ para que se tenha um menor risco de desenvolver a incapacidade funcional é necessário uma boa aptidão física, pois a mesma encontrasse relacionada com boa saúde. Essas aptidões são postas à prova com as atividades do dia-a-dia, como levantar-se, desviar de obstáculos, andar pela casa, entre outras atividades cotidianas, com o decrescimento das aptidões como a força, a agilidade, flexibilidade e coordenação, o idoso apresentará alguma dificuldade para realizar mais de um movimento ao mesmo tempo, acarretando assim mais dificuldade para este idoso poder realizar suas atividades cotidianas.

A literatura tem mostrado que idosos que praticam alguma atividade física regulamente, tem aumento da capacidade funcional, e assim apresentam melhor desempenho nas suas AVD's, além de terem maior disposição e uma qualidade de vida significativa em comparação a idosos sedentários, por tanto é de fundamental importância que os idosos pratiquem atividades físicas para que sua capacidade funcional se mantenha em bom estado e assim tornem-se mais participativos ${ }^{4}$.
Barbosa et $\mathrm{al}^{1}$ afirma que há maior prevalência nas Atividades Instrumentais de Vida Diária-AIVD, do que nas Atividades Básicas da Vida diária-ABVD, as perdas ocorrem de atividades instrumentais de vida diária para atividades básicas de vida diária, pois as AIVD exigem maior integridade física cognitiva comparadas às $A B V D$, entretanto outros estudos nos mostram o contrário, por isso é muito importante que o idoso esteja sempre realizando suas tarefas do seu cotidiano para que seu cognitivo esteja sempre funcionando e consequentemente se encontrem em perfeito estado.

Para o idoso ter sua capacidade funcional preservada, ele deve estar envolvido socialmente, participando das atividades sociais, tendo lazer adequadamente, sendo motivado a fazer suas atividades diárias, desta forma mantem seu cognitivo preservado. Estar envolvido socialmente e ter satisfação, sentir-se útil é importante para que se tenha um envelhecimento saudável, pois são elementos que contribuem para que não haja isolamento do idoso que acarreta a depressão $0^{5}$. Diante deste contexto, a hipótese deste estudo é verificar se há comprometimentos quanto a autonomia funcional em idosos comunitários.

A fisioterapia em gerontologia tem sido destaque nos níveis primário e secundário, na saúde da crescente população idosa. Considerando que 0 idoso tende a ter um declínio gradativo em suas funções clínicas, torna-se este estudo relevante, que tem como objetivo avaliar a autonomia funcional em idosos comunitários.

\section{Materiais e métodos}

Caracteriza-se como uma pesquisa de campo tipo descritiva, observacional, e transversal, realizada no mês de setembro de 2017, com 34 idosos comunitários de ambos os sexos, com idade igual ou 
superior a 60 anos na União de Moradores do bairro Sol e Mar, do município de São Luís, no estado do Maranhão. Foram incluídos apenas idosos com idade igual ou superior a 60 anos, e excluídos os idosos que não são vinculados a União de Moradores do bairro Sol e Mar.

Foi utilizado o Questionário validado criado pelo Grupo de Desenvolvimento Latino-Americano para a MaturidadeGDLAM $^{6}$, que consiste em:

Caminhar 10 metros com o propósito deste teste é avaliar a velocidade que o indivíduo leva para percorrer a distância de 10 metros (C10m).

(LPS): Levantar-se da posição sentada esse teste visa avaliar a capacidade funcional da extremidade inferior e consiste em: o indivíduo, partindo da posição sentada em uma cadeira, sem apoio dos braços, estando o assento a uma distância do solo de $50 \mathrm{~cm}$, levantarse e sentar-se, cinco vezes consecutivamente.

(LPDV): Levantar-se da posição decúbito ventral com o propósito deste teste é avaliar a habilidade do indivíduo para levantar-se do chão. O teste consiste em: partindo da posição inicial em decúbito ventral com os braços ao longo do corpo, ao comando de "já", o indivíduo deve levantar-se, ficando de pé o mais rápido possível. (LCLC): Levantar-se da cadeira e locomover-se pela casa - o objetivo é avaliar a capacidade do idoso na sua agilidade e equilíbrio, em situações da vida. Com uma cadeira fixa no solo, devem-se demarcar dois cones diagonalmente à cadeira, a uma distância de quatro metros para trás e de três metros para os lados direito e esquerdo da mesma. $O$ indivíduo inicia o teste sentado na cadeira com os pés fora do chão, e ao sinal de "já", ele se levanta, move-se para direita, circula o cone, retorna para a cadeira, senta-se e retira ambos os pés do chão. Sem hesitar faz o mesmo movimento para a esquerda. Imediatamente, realiza novo percurso, para a direita e para a esquerda, assim perfazendo todo 0 percurso e circulando cada cone duas vezes em menor tempo possível.

(VTC): O indivíduo deve estar de pé com os braços ao longo do corpo e com uma camiseta em uma das mãos. Ao sinal de "já", ele deve vestir a camiseta e, imediatamente, retirá-la, retornando a posição inicial. Este visa avaliar a agilidade e a coordenação dos membros superiores.

A fórmula para o cálculo do índice GDLAM (IG) é: $\mathrm{IG}=(\mathrm{C} 10 \mathrm{~m}+\mathrm{LPS}+\mathrm{LPDV}+\mathrm{VTC}) \times 2+(\mathrm{LCLC})$ : 4, onde C10m, LPS, LPDV e LCLC=tempo aferido em segundos, e $\mathrm{IG}=$ Índice GDLAM em escores.

Os valores obtidos no GDLAM (IG) classificam a autonomia funcional em: Fraco (>27,42), Regular (de 24,98 a $27,42)$, Bom $(22,66$ a $<24,98)$, Muito bom $(<22,66)$.

O presente estudo foi aprovado pelo Comitê de Ética em Pesquisa em Humanos (CEP), da Universidade CEUMA, sob parecer no 289.169/2013.

Os dados foram digitados e analisados no SPSS 18.0. Na estatística descritiva, as variáveis qualitativas são apresentadas através de frequências absolutas e relativas. Para comparar a autonomia funcional por sexo e faixa etária foi utilizado o Qui-quadrado sendo considerado significativo $p \leq 0,05$.

\section{Resultados}

Foram inseridos no estudo 34 idosos, sendo $91,2 \%$ (31) do sexo feminino e $8,8 \%$ (3) masculino. Entre os analisados, 55,9\% (19) tem entre 60 e 70 anos e 44,1\% (15) entre 71 e 81 anos. A. Na avaliação com o protocolo do Grupo de Desenvolvimento Latino-Americano para a Maturidade (GDLAM) foi verificado que 85,3\% (29) dos idosos da amostra apresentam capacidade funcional fraca (gráfico 1). 
$\mathrm{Na}$ comparação da autonomia funcional por sexo foi verificado que entre as idosas $83,9 \%$ apresentam autonomia fraca e entre os homens $100 \%$ (gráfico 2). O valor do Qui-quadrado foi de 0,567 com uma possibilidade associada ( $p$-valor) de 0,753 , mostrando que a diferença de frequência é bastante provável apenas como resultado do erro amostral (ao acaso).

Com relação à faixa etária, embora tenha sido observado que $89,5 \%$ dos idosos com idade entre 60 e 70 anos tenham autonomia funcional fraca e entre aqueles com idade entre 71 e 81anos apenas $80,0 \%$ tenham autonomia fraca (gráfico 3), essa diferença não mostrou-se estatisticamente significativa $(p=0,494)$.

\section{Discussão}

Identificaram-se no presente estudo que os idosos comunitários apresentam baixa capacidade funcional, sendo $85,3 \%$ fraca, 11,8\% regular e apenas 2,9\% bom. Esses resultados podem ter sido gerados porque grande parte dos idosos apresentaram algum tipo de patologia que comprometia membros superiores ou inferiores, o que acabou impossibilitando que os mesmos fizessem 0 teste do GDLAM em menor tempo, já que o teste é todo dado em tempos, portanto não obtiveram um resultado satisfatório. Foi observado que 83,9 \% das mulheres apresentaram autonomia fraca para $100 \%$ dos homens.

Um estudo realizado por Assis et al ${ }^{7}$ mostrou resultados semelhantes com os encontrados neste artigo, seus resultados mostram os idosos com capacidade funcional não adequada, sua amostra foi composta por (175) idosos, dos quais $68,5 \%$ (120) eram mulheres e 31,5\% (55) eram homens, sua análise mostrou uma diminuição da capacidade funcional, quando associada a idade. Já Garcia et al
8 realizou estudo com 59 idosos, onde $52,6 \%$ apresentaram IG muito bom, 7,1\% regular e $22,8 \%$ fraco, todos os idosos que participaram do seu estudo eram ativos, 0 que implica dizer que para apresentar uma boa capacidade funcional é necessário ter uma vida ativa.

Machado et $\mathrm{al}^{9}$ em estudo com idosos com idade de 81,0 \pm 7,1 anos, foi apresentado que a maioria dos idosos tem dificuldade grave nas atividades de cuidados pessoal com exceção para as tarefas como comer, beber e cuidados com o processo de excreção. Os idosos se apresentaram bastante prejudicados com a autonomia para resolverem problemas, realizar rotinas diárias, participação na vida comunitária, associações informais e socialização.

As mulheres apresentaram a capacidade funcional melhor que os homens participantes desse estudo, que foram apenas 03 , os três relataram que sentiam dores em membros superiores ou inferiores, 0 que comprometeu 0 desempenho. Já em outro estudo realizado por Pinto et al ${ }^{5}$ as mulheres apresentaram desvantagens em relação a capacidade funcional, pois com base em estatísticas as mulheres vivem cerca de 8 a 10 anos mais do que homens, dessa forma apresentaram mais vulnerabilidade a terem doenças crônicas não transmissíveis, o que implica dizer que necessitaram de cuidados e ajuda para realizarem suas atividades de vida diária.

Este estudo mostrou que idosos entre 71-81 anos tiveram resultados melhores do que aqueles com idade entre 60-70 anos, observou-se que os idosos de idade mais avançada realizam os testes com mais vigor do que os idosos mais novos, que fez com que neste estudo idoso longevos entre 71-81 anos tivessem resultados melhores quanto a autonomia funcional do que aqueles entre $60-70$ anos que são 


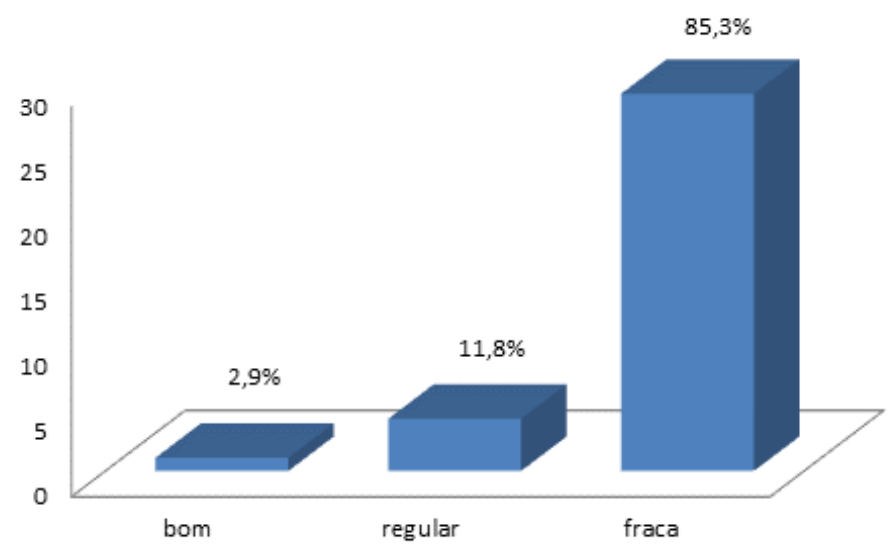

Gráfico 1 - Autonomia funcional de idosos comunitários. São Luís- MA, 2017.

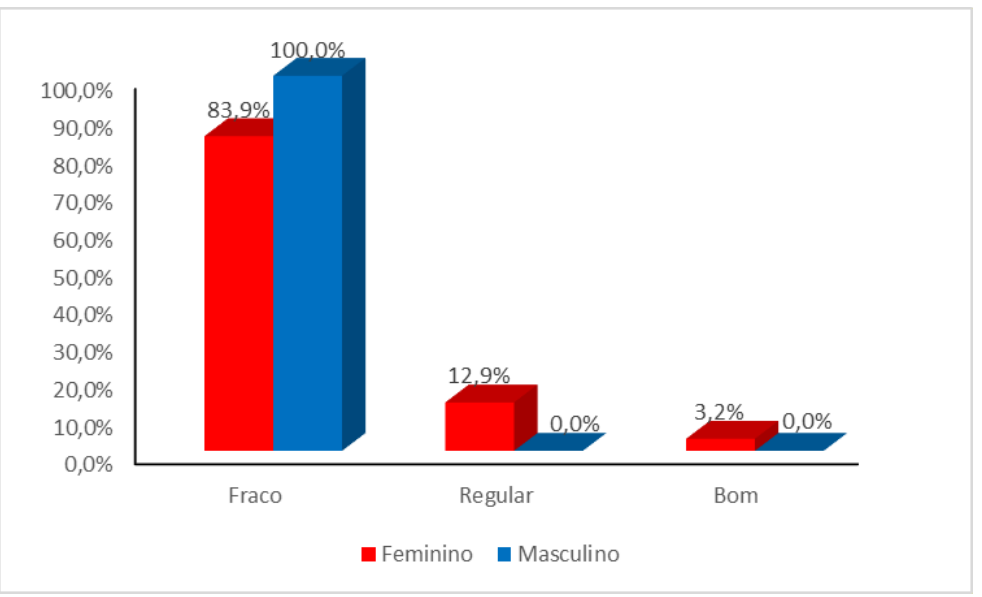

Gráfico 2 - Comparação da autonomia funcional de idosos comunitários por sexo. São LuísMA, 2017.

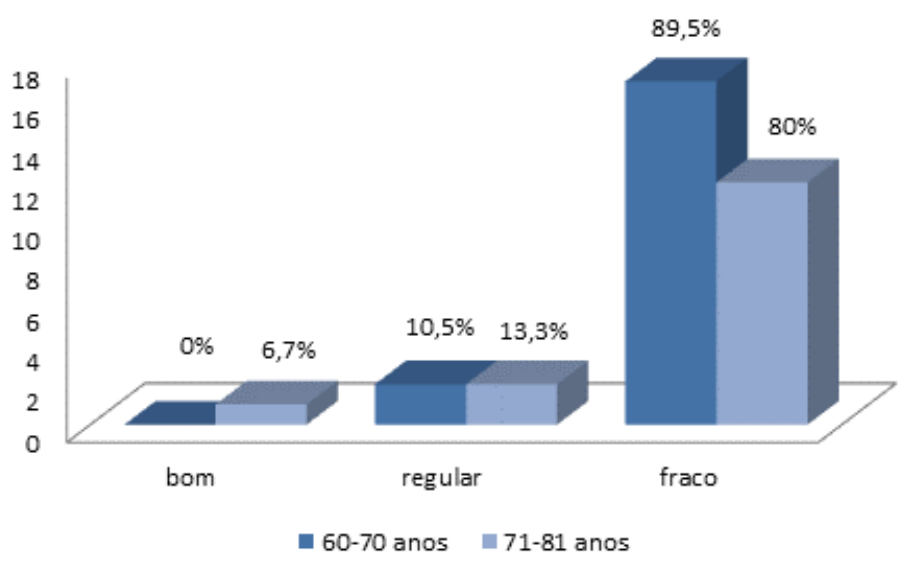

Gráfico 3 - Comparação da autonomia funcional de idosos comunitários por faixa etária. São Luís- MA, 2017 
idosos mais novos. Maia et al ${ }^{10}$ mostra no seu estudo que a maioria dos idosos apresentaram elevada independência para realizarem grande parte das AVDs, os indivíduos mais idosos apresentaram maior nível de dependência sendo que aqueles que possuem pior percepção sobre seu estado de saúde apresentam maior dependência funcional.

Andriolo et al ${ }^{4}$ mostrou em estudo que de 165 idosos que participaram da sua amostra, 69,7\% apresentaram resultados equivalente a independência, e 30,3\% apresentam dependência parcial, nenhum dos idosos do estudo apresentaram dependência total em suas AVDs. Idosos com idade média de 72,24 \pm 9,33 anos na avaliação da capacidade funcional identificou-se que a maioria realizava suas atividades de vida diária (AVDs) e as atividades instrumentais de vida diária (AIVDs) de forma independente, os idosos do seu estudo são na maioria independentes, apresentando um envelhecimento bem-sucedido. Um estudo com 134 idosos mostrou que para realizarem atividades básicas de vida diária $129(66,4 \%)$ idosos mostraram-se independentes e $5(3,7 \%)$ dependentes, já nas atividades instrumentais de vida diária $78(58,2 \%)$ apresentaram independência e $56(41 \%)$ dependência (PASSATO et al ${ }^{11}$ ).

Freitas et al ${ }^{12}$ mostra que os idosos participantes do seu estudo, tiveram boa autonomia funcional, e que a maioria relatou saúde regular $(62,1 \%)$ seguida de boa $(18,4 \%)$ e ruim $(16,5 \%)$, $(2,9 \%)$ relataram saúde excelente e não houve relatos de saúde péssima. O que nos mostra que a saúde interfere diretamente na autonomia funcional. Estudo realizado por Alencar et al ${ }^{13}$ que comparava idosas ativas e não ativas, mostrou que as idosas ativas, obtiveram resultados melhores do que as não ativas, mas quando os valores eram comparados ao do GDLAM, os dois grupos apresentaram IG fraco.
Os idosos que participaram deste estudo faziam atividades recreativas como artesanato, alongamento, mas não tratavam suas patologias, artrose, artrite entre outras comodidades relatadas. Muitos idosos ainda relataram ter contraído Zika vírus, e que por decorrência disso sentiam dores articulares. Um estudo realizado por Ruzene ${ }^{14}$ aponta que idosas participantes de EF, seja ele aeróbico ou resistido apresentam melhor mobilidade que aquelas que não participam, as idosas eram independentes nas atividades de vida diária e fisicamente ativas.

Idosos que praticam atividades físicas, que não possuem doenças, que estão inseridos no convívio social, irão apresentar autonomia boa para realizarem suas tarefas de vida diária, o que não foi possível verificar neste estudo, pois grande parte dos idosos participantes relataram apresentar algum tipo de dor, causada por patologias adquiridas ao longo da vida ou quedas, mesmo muitos ainda realizando com muita dificuldade suas AVD's.

\section{Conclusão}

Concluiu-se que a grande maioria da amostra estudada apresentou níveis baixos de autonomia funcional, em especial nos idosos com menor faixa etária. $O$ fato de grande parte não praticar algum tipo de atividade física pode ter contribuído para tais achados.

A amostra avaliada vive de forma independente na sociedade e o nível de fragilidade se apresenta como fator influenciador para a continuidade dessa independência. Dessa forma o presente estudo contribui para o despertar em relação à importância de intervenções que busquem melhora da autonomia funcional da população idosa. 


\section{Referências}

1. Barbosa, R. et al. Avaliação da capacidade funcional dos idosos e fatores associados a incapacidade. Revista Ciência \& Saúde Coletiva; 2014; 19(8):3321-3322.

2. Freitas, R.S. et al. Capacidade funcional e fatores associados em idosos: Estudo populacional, Revista Acta Paulista Enfermagem; 2012; 25(6):937.

3. Gonçalves, L.H.T. et al. O idoso institucionalizado: Avaliação da capacidade funcional e aptidão física, Revista Caderno de Saúde Publica; 2010; 26(9):1742.

4. Andriolo, B.N.G. et al. Avaliação do grau de funcionalidade em idosos usuários de um centro de saúde. Revista Sociedade Brasileira de Clínica Medicina; 2016; 14(3):142.

5. Pinto, J.M. et al. Doenças crônicas, capacidade funcional, envolvimento social e satisfação em idosos comunitários: estudo fibra, Revista Ciência \& Saúde Coletiva; 2013; 18(12):3458.

6. Dantas, E. E. M.; VALE, R. G. S. Protocolo GDLAM na Avaliação da Autonomia Funcional. Fitness \& Performance Journal, Rio de Janeiro; 2004; 3(3):175-183.

7. Assis, V.G. et al. Prevalência e fatores associados a capacidade funcional de idosos na estratégia saúde da família de Monte Claros, Minas Gerais Brasil.
Revista Brasileira de Geriatria e Gerontologia; 2014; 17(1):158.

8. Garcia, J.S et al. Avaliação da autonomia funcional do idoso ativo e sedentário. Revista Cientifica FUNVIC; $2016 ; 1(1): 65$..

9. Machado, F.N. et al. Comparação entre a capacidade e desempenho: um estudo sobre funcionalidade de idosos dependentes. Revista Latino-Am. Enfermagem; 2013; 21(6):1324.

10. Maia, L. et al. A funcionalidade como determinante do envelhecimento ativo. INFAD Revista de Psicologia; 2016; 1(2):229.

11. Passatto, J. M. et al. Condições de saúde psicologica, capacidade funcional e suporte social de idoso. Revista Kairós - Gerontologia; 2017; 20(2):51.

12. Freitas, C.V. et al. Avaliação de fragilidade, capacidade funcional $e$ qualidade de vida dos idosos atendidos no ambulatório de geriatria de um hospital universitário. Revista Brasileira de Geriatria e Gerontologia; 2016; 19(1):124, 2016.

13. Alencar, N.A. et al. Nível de atividade física, autonomia funcional e qualidade de vida em idosas ativas e sedentárias. Revista Fisioter Mov; 2010; 23(3):478.

14. Ruzene, J.R.S. et al. Avaliação do equilíbrio, mobilidade e flexibilidade em idosas ativas e sedentárias. Revista Brasileira de Geriatria e Gerontologia; 2014;17(4):790. 